\title{
Surface-based Map Plasticity of the Sensorimotor Cortex in Hip Disorder at Local and Extensive Levels: A Resting-state fMRI Study
}

Jie Ma

School of Rehabilitation Science

\section{Xu-Yun Hua}

Department of Traumatology and Orthopedics

\section{Mou-Xiong Zheng}

Department of Traumatology and Orthopedics

Jia-Jia Wu

Center of Rehabilitation Medicine

\section{Bei-Bei Huo}

School of Rehabilitation Science

\section{Xiang-Xin Xing}

School of Rehabilitation Science

\section{Sheng-Yi Feng}

Department of Traumatology and Orthopedics

\section{Bo Li}

School of Rehabilitation Science

Jian-Guang Xu ( $\nabla$ xjg@shutcm.edu.cn )

School of Rehabilitation Science Shanghai University of Traditional Chinese Medicine, Shanghai, China

\section{Research}

Keywords: hip disorder, Surface-based Map Plasticity, ReHo, FC, cortical thickness

Posted Date: October 9th, 2020

DOI: https://doi.org/10.21203/rs.3.rs-87128/v1

License: (c) (i) This work is licensed under a Creative Commons Attribution 4.0 International License.

Read Full License 


\section{Abstract}

Background: Pain is one of the manifestations of hip disorder and has been proven to lead to the remodeling of somatotopic map plasticity in the cortex. However, it's not clear whether hip disorder with pain induces somatotopic map plasticity in the cortex. We aimed to evaluate the surface-based map plasticity of the somatotopic cortex in hip disorder at local and extensive levels by resting-state functional magnetic resonance imaging ( $\mathrm{rs}-\mathrm{fMRI})$.

Methods: 20 patients with osteonecrosis of the femoral head (ONFH) (12 males and 8 females, age= $56.80 \pm 13.60$ years) with Visual Analogue Scale (VAS) scores $\geq 4$ and 20 healthy controls (9 males and 11 females, age $=54.56 \pm 10.23$ years) were enrolled in this study. rs-fMRI data and T1 imaging data were collected, and surface-based regional homogeneity (ReHo), seed-based functional connectivity (FC), cortical thickness and the volume of subcortical gray nuclei were calculated.

Results: Compared with the healthy controls, the ONFH patients showed significantly increased surfacebased ReHo in areas distributed mainly in the left dorsolateral prefrontal cortex and frontal eye field, the right frontal eye field and the premotor cortex and decreased surface-based ReHo in the right primary motor cortex and primary sensory cortex. When the area with decreased surface-based ReHo in the frontal eye field and right premotor cortex was used as the regions of interest (ROI), compared with the controls, the $\mathrm{ONFH}$ patients displayed increased FC in the right middle frontal cortex and right inferior parietal cortex and decreased FC in the right precentral cortex and right middle occipital cortex. ONFH patients also showed significantly decreased cortical thickness in the para-insular area, supplementary motor cortex area and frontal eye field and decreased volume of subcortical gray matter nuclei in the right nucleus accumbens $(479.32 \pm 88.26$ vs $539.44 \pm 68.36, P=0.026)$.

Conclusions: Hip disorder patients showed cortical plasticity changes, mainly in sensorimotor and painrelated regions.

\section{Background}

Osteonecrosis of the femoral head (ONFH), also known as avascular or ischemic necrosis of femoral head, is a hip disorder and challenging orthopedic disease that severely diminishes patient quality of life. The risk factors of ONFH are varied and include physical trauma, smoking, excessive alcohol consumption, autoimmune diseases, coagulation disorders, hemoglobinopathies, corticosteroid therapy and other factors ${ }^{1}$. ONFH is a progressive pathological process characterized by ischemia, necrosis, and eventually collapse. Despite no predominant pattern of a clinical presentation, pain and dysfunction of the joint seem occur frequently in $\mathrm{ONFH}^{2}$. Pain presents in only half of patients with no limitation of activity in the early stages but almost always appears in later stages ${ }^{2}$. The disease generally progresses to collapse and eventually painful osteoarthritis if symptomatic ONFH has not been treated ${ }^{3}$. The treatment strategy of ONFH depends on the disease stage. Reducing pain is one of the main goals of early treatment ${ }^{4}$. Total hip arthroplasty (THA) in patients with osteonecrosis is widely used to improve 
patient quality of life. However, revision surgeries after THA in young patients are common, and thigh pain is a common complaint after $\mathrm{THA}^{5}$. This pain is a primary factor influencing patients' dissatisfaction after $\mathrm{THA}^{6}$.

As observed in $\mathrm{ONFH}$, joint diseases cause pain, and the impaired joints and surrounding muscles, ligaments and tendons can cause proprioception abnormalities. Such abnormal sensory perception leads to the remodeling of the sensory cortex. The mature human primary somatosensory cortex can reorganize itself in response to changes in sensory input, displaying strong plasticity capacity. Following the elimination of afferent return, there is a well-known 'invasion' of the deafferented region of the brain by the cortical representation zones of still-intact portions of the brain adjacent to it ${ }^{7}$. Human and animal studies have confirmed that sensory deprivation can induce somatotopic map plasticity in the primary somatosensory cortex ${ }^{7,8}$. Prolonged and chronic pain can both alter neural plasticity at the cortical level ${ }^{9}$. It has gradually been recognized that cerebral processes contribute to pain beyond the level of nociceptive input and contact behavioral and psychological influences ${ }^{10}$. Pain is a complex sensory and emotional experience that is shaped by psychobiology, expectations from past and learned pain experiences and attention processes ${ }^{11}$. In a study of intestinal diseases related to abdominal pain, researchers found altered neural modulation of pain in affective and cognitive brain regions, including the cingulate cortex ${ }^{12}$. Brain regions of headache patients showed significant activations in areas responsible for the processing of cognitive empathy ${ }^{13}$. Current theories describe the brain activity of pain as abnormal functioning in large-scale networks that include non-nociceptive regions ${ }^{14,15}$. Chronic cervical spondylotic pain, chronic back pain and knee osteoarthritis show functional anomaly during the pain process ${ }^{16,17}$. Subjects with cruciate ligament deficiency also exhibit increased activation in the secondary somatosensory area (where pain and sensory processing occur) ${ }^{18}$.

The remodeling of somatosensory cortex evidences the plasticity of the somatotopic map ${ }^{19}$. Recently, somatosensory cortex stimulation has been proposed as a possible treatment for deafferentation pain after amputation ${ }^{20}$. Exploring the map plasticity in patients with ONFH can provide insight into its possible treatment. Therefore, based on previous studies that have discovered abnormal brain activity in many kinds of diseases related to sensory abnormality, we hypothesized that ONFH with pain may induce somatotopic map plasticity in cortex and yield a characteristic pattern of brain neural activity. Volumebased normalization may introduce inaccuracies in anatomical positioning of functional data 21,22 , and it is difficult to account for inter-subject variability in gyrus size, shape or position in a 3D referential; furthermore, such differences may correspond to the displacement of a functional focus to a different gyrus in some subjects ${ }^{22}$. As a large part of the data originates from the cortex, methods that work on the cortical surface may be more sensitive than those using the full brain volume and thus be more suitable for map plasticity study.

Diverse approaches can be used to detect map plasticity, including genetics analysis, synaptic and in vivo physiology analysis, optical imaging, and ultrastructural analysis ${ }^{19}$. Although these methods show excellent validity and reliability, they are not simple or convenient to use in the human body. An article 
published in the New England Journal of Medicine reported increased activity in the stable patterns across regions that are associated with experimentally induced acute pain and tonic pain in healthy people. They proposed that a functional magnetic resonance imaging (fMRI) could be used to assess pain ${ }^{23}$. Resting-state functional magnetic resonance imaging rs-fMRI (rs-fMRI), as a convenient and noninvasive method, has been widely used in the evaluation of central remodeling in various diseases related to pain. Therefore, in our study, we chose fMRI as the assessment tool to test our hypothesis. To comprehensively understand the characteristics of somatotopic map plasticity in patients with $\mathrm{ONFH}$, an understanding of the roles of pain-related brain regions and functional connections is needed. Therefore, among several functional measures of $\mathrm{fMRI}$, surface-based regional homogeneity ( $\mathrm{ReHo})^{24}$ and seedbased functional connectivity $(F C)^{25}$ were chosen to analyze localized and remote changes in brain functions. Compared with 3D ReHo, surface-based ReHo has higher test-retest reliability and can more clearly reveal the intrinsic functional organization of cortex ${ }^{26}$, which could be extremely useful for integrating various measures of both the structure and function of the cortical surface ${ }^{27}$. Cortical thickness and the volume of subcortical gray nuclei were also analyzed in the study.

\section{Materials And Methods}

Clinical Samples

A total of 20 right-handed ONFH inpatients without surgery (12 males and 8 females, age $=56.80 \pm 13.60$ years) who were consecutively recruited and 20 right-handed healthy controls ( 9 males and 11 females, age $=54.56 \pm 10.23$ years) were enrolled in this study. Diagnosis of ONFH was made based on magnetic resonance imaging (MRI) and clinical demonstrations. Patients were included if they had joint pains in groin, buttock and thigh areas and Visual Analogue Scale (VAS) scores for pain ${ }^{28} \geq 4$ over 1 year. Individuals were excluded from the study if they had a history of cardiovascular or cerebrovascular disease, ankylosing spondylitis, rheumatoid arthritis, hip dysplasia, metabolic disorders, or bone tumor. Informed consent was obtained from all participants included in the study. There was no statistical difference in background data between ONFH patients and healthy controls, including education, past history and operation history.

MRI Data Acquisition

MRI data were acquired using a MAGNETOM Verio 3.0 T scanner (Siemens Healthcare, Germany).

1. Functional imaging

rs-fMRI data were obtained with eye closed and a single-pass gradient recalled EPI sequence with the following protocols: interleaved scanning order, slice number $=43$, flip angle $=90^{\circ}$, matrix size $=64 \times 64$, $\mathrm{TR}=3000 \mathrm{~ms}$, slice thickness $=3.0 \mathrm{~mm}, \mathrm{FOV}=230 \times 230 \mathrm{~mm}^{2}$, gap $=0$ (voxel size $3.6 \times 3.6 \times 3.0 \mathrm{~mm}^{3}$ ), and number of acquisitions $=200$.

2. Structural imaging 
T1-weighted magnetization-prepared rapid acquisition was performed with the following parameters: repetition time/inversion time/echo time $=1900 / 900 / 2.93 \mathrm{~ms}$, flip angle $=9^{\circ}, \mathrm{FOV}=256 \times 256 \mathrm{~mm}^{2}$, section thickness $=1.0 \mathrm{~mm}$ sagittal acquisition, acquisition matrix $=256 \times 256$, number of averages $=1$.

\section{Data processing}

Functional and structural images of each subject were preprocessed by using DPABI ${ }^{29}$ (DPARSurf V4.3), which is based on Statistical Parametric Mapping (SPM12) (http://www.fil.ion.ucl.ac.uk/spm), on a MATLAB 2013b platform. Preprocessing was performed as previously reported ${ }^{30}$. (1) slice scan time correction; (2) head movement correction (the head movements were all less than $2.5 \mathrm{~mm}$ or 2.5 degrees in any direction); (3) spatial normalization; (4) regression of nuisance variables (the white matter and cerebral spinal fluid blood oxygen level dependent (BOLD) signal and the effects of head motion using six head motion profiles). Then, ReHo and FC were calculated for the traditional low frequency band $(0.01-0.10 \mathrm{~Hz})$. Spatial smoothing (FWHM $=6 \mathrm{~mm}$ ) was performed after surface-based ReHo calculation as in previous studies ${ }^{30}$. Regions showing significant differences in surface-based ReHo values between the two groups were defined as regions of interests (ROIs) for seed-based FC analysis to investigate interregional functional synchronization, and FC calculation was performed as in previous studies ${ }^{31}$.

Statistical analysis

Two-sample t-tests were performed by using SPSS to evaluate differences in the volume of subcortical gray nuclei between ONFH patients and healthy controls. The level of two-tailed statistical significance was set at $P<0.05$. Two-sample t-tests were used for analyses of surface-based ReHo and cortical thickness differences between the ONFH patients and healthy controls. The results were corrected for multiple comparisons, with a combined threshold of a single voxel $(P<0.05)$. A more strict threshold $(P<$ 0.01 , cluster size $>50$ voxels) was also used for each cohort to reduce the possibility of false negative results. Two-sample t-tests were used to analyses FC differences between the ONFH patients and healthy controls. We employed a cluster-level family-wise error (FWE) correction for multiple comparisons (P区 0.05).

Sample size calculation

This is a cross-sectional study. The essence of fMRI image is digit, fMRI data is measurement data. We planned a study of a continuous variable from independent control subjects (healthy controls) and experimental subjects (ONFH). Considering a true difference in the experimental and control means of 0.07 in ReHo, we needed to study at least 18 experimental subjects and 18 control subjects to be able to reject the null hypothesis that the population means of the experimental and control were equal with probability (power) 0.80 . The type I error probability associated with this test of this null hypothesis was $0.05(a)$.

\section{Results}




\section{Comparison of functional images between ONFH patients and healthy controls}

Compared to healthy controls, ONFH patients showed significantly increased surface-based ReHo in areas distributed mainly in the left dorsolateral prefrontal cortex and frontal eye field, the right frontal eye field and premotor cortex and decreased surface-based ReHo in the right primary motor cortex and primary sensory cortex (Figure 1 and Table 1).

Regions showing significant differences in surface-based ReHo values between the two groups were defined as ROls for seed-based FC analysis. As shown in Figure 2, when the area with decreased surfacebased ReHo in the right frontal eye field and premotor cortex was used as ROI, the ONFH patients displayed increased FC in the right middle frontal cortex and right inferior parietal cortex and decreased FC in the right precentral cortex and right middle occipital cortex (Figure 2 and Table 2). FC values of another two ROls did not significantly differ between the two groups.

\section{Comparison of structural images between ONFH patients and healthy controls}

Compared to healthy controls, ONFH patients showed significantly decreased cortical thickness in areas mainly distributed in the para-insular area, posterior insular area, anterior superior temporal area, frontal eye field and supplementary motor cortex. In addition, comparison of the volume of subcortical gray matter nuclei between the two groups revealed significantly decreased values in the right nucleus accumbens in ONFH patients compared with healthy controls (479.32 \pm 88.26 vs $539.44 \pm 68.36, P=0.026$; Figure 3 and Table 3).

Table 1: Comparison of surface-based ReHo values between ONFH patients and healthy controls

\begin{tabular}{|c|c|c|c|c|}
\hline $\begin{array}{l}\text { Overlap of atlas } \\
\text { region }\end{array}$ & Hemisphere & $\begin{array}{l}\text { Cluster } \\
\text { size }\end{array}$ & $\begin{array}{l}\text { Cluster centroid MNI } \\
\text { Coordinates }\end{array}$ & $\begin{array}{l}\text { Peak } t \\
\text { value }\end{array}$ \\
\hline
\end{tabular}

$$
\begin{array}{lll}
X & Y & Z
\end{array}
$$

$42.86 \%$ dorsolateral prefrontal cortex $(9-46 \mathrm{~d})$

L $\quad 55.62$

6.28

79.50

28.10

3.66

$28.57 \%$ frontal eye field $(8 \mathrm{Ad})$

$28.57 \%$ dorsolateral prefrontal cortex(9p)

$88.89 \%$ frontal eye field(8Av)

$\mathrm{R}$

67.25

6.28

52.78

38.68

4.09

$11.11 \%$ premotor cortex and frontal eye field(i6-8)

$95 \%$ primary sensory cortex $(3 a, 3 b)$

R $\quad 56.91$

$-14.74$

$-12.42$

70.83

$-4.01$

$5 \%$ primary motor cortex $(4)$ 
Table 2: Comparison of FC values between ONFH patients and healthy controls

\begin{tabular}{|c|c|c|c|c|c|}
\hline \multirow[t]{2}{*}{ Region Label } & \multirow[t]{2}{*}{ Cluster size } & \multicolumn{3}{|c|}{ Cluster centroid MNI Coordinates } & \multirow[t]{2}{*}{$t$-value } \\
\hline & & $x$ & $\mathrm{Y}$ & Z & \\
\hline right middle frontal cortex & 2233 & 42 & 23 & 53 & 9.12 \\
\hline right inferior parietal cortex & 829 & 50 & -55 & 52 & 7.23 \\
\hline right precentral cortex & 999 & 15 & -27 & 65 & -9.25 \\
\hline right middle occipital cortex & 202 & 42 & -74 & 3 & -6.90 \\
\hline
\end{tabular}

Table 3: Comparison of cortical thickness between ONFH patients and healthy controls

\begin{tabular}{|c|c|c|c|c|c|c|}
\hline \multirow[t]{2}{*}{ Overlap of atlas region } & \multirow[t]{2}{*}{ Hemisphere } & \multirow[t]{2}{*}{$\begin{array}{l}\text { Cluster } \\
\text { size }\end{array}$} & \multicolumn{3}{|c|}{$\begin{array}{l}\text { Cluster centroid MNI } \\
\text { Coordinates }\end{array}$} & \multirow{2}{*}{$\begin{array}{l}\text { Peak } \\
t \\
\text { value }\end{array}$} \\
\hline & & & $X \quad Y$ & Z & & \\
\hline 64.89\%para-Insular area(PI) & \multirow[t]{3}{*}{$\mathrm{R}$} & \multirow[t]{3}{*}{79.73} & \multirow[t]{3}{*}{20.43} & \multirow[t]{3}{*}{27.12} & \multirow[t]{3}{*}{-38.44} & \multirow[t]{3}{*}{-3.48} \\
\hline $24.04 \%$ posterior Insular area(Pol1) & & & & & & \\
\hline $\begin{array}{l}11.07 \% \text { anterior superior temporal } \\
\text { area(TA2) }\end{array}$ & & & & & & \\
\hline $\begin{array}{l}70.65 \% \text { supplementary motor cortex } \\
\text { and frontal eye field } \llbracket \text { s6-8 } 8\end{array}$ & \multirow[t]{2}{*}{$\mathrm{R}$} & \multirow[t]{2}{*}{67.69} & \multirow[t]{2}{*}{-19.30} & \multirow[t]{2}{*}{54.03} & \multirow[t]{2}{*}{53.80} & \multirow[t]{2}{*}{-4.14} \\
\hline $\begin{array}{l}29.35 \% \text { supplementary motor cortex } \\
\rrbracket 6 \mathrm{ma} \rrbracket\end{array}$ & & & & & & \\
\hline
\end{tabular}

\section{Discussion}

In this study, we revealed differences in functional and structural imaging between ONFH patients and healthy controls that may reveal the characteristics of altered central pain processing in ONFH patients. The brain regions exhibiting differences between the two groups included the dorsolateral prefrontal cortex, frontal eye field, premotor and supplementary motor cortex, primary motor cortex, primary sensory cortex, middle frontal cortex, inferior parietal cortex, precentral cortex, middle occipital cortex, insula and 
nucleus accumbens. These regions are mainly sensorimotor and pain-related regions. Our functional and structural analyses both support previous neuroimaging findings regarding pain and provide novel findings that may provide the foundation for future larger studies in $\mathrm{ONFH}$.

Pain is one symptom of ONFH and is usually confined to the groin area, occasionally involving the ipsilateral hip and knee or greater trochanteric area ${ }^{32}$. There are two complementary pathways related to pain processing: the medial and lateral pain pathways ${ }^{33}$. In the present study, ONFH patients showed functional decline in the primary sensory cortex, which is compatible with the existence of complementary pathway related to pain processing and align with the results of brain function studies in other non-central nervous system diseases with pain ${ }^{34,35}$. Interestingly, both of these pathways involve the insular cortex. In the pain matrix, the insular cortex is mainly involved in discriminative sensory and motivative emotion. Abnormal signal transmission from the injury site causes neuropathic pain, which generates enhanced synaptic plasticity ${ }^{36}$. Our study showed decreased cortical thickness of the insular cortex, which is consistent with the findings of other pain studies in chronic migraine, cervical spondylosis with neck pain and irritable bowel syndrome $\mathrm{e}^{37-39}$. Usui $\mathrm{C}$ et al ${ }^{40}$ observed that patients with fibromyalgia showed a significant difference in connectivity between the insular cortex and other brain regions. After analgesic treatment, the insular cortex is activated in low back pain ${ }^{41}$ and fibromyalgia ${ }^{40}$. We also observed that the volume of the nucleus accumbens was significantly decreased in the ONFH patients compared with the healthy controls. Makary $\mathrm{MM}$ et al ${ }^{42}$ provided evidence that a lower nucleus accumbens volume confers risk for developing chronic pain and that altered nucleus accumbens activity is a signature of the state of chronic pain.

Numerous studies have demonstrated that pain processing can shift from nociceptive somatosensory pathways to emotional brain circuits ${ }^{43,44}$. Increased activation of the prefrontal cortex (PFC) is related to decreased pain and inhibits the functional connectivity between the midbrain and the medial thalamus ${ }^{45}$. Further projections from the anterior cingulate cortex to the PFC may also be involved in cognitive appraisal of the stimulus. In addition, prefrontal responses to pain depend on the psychological state of the subject, who may expect worse pain or a reduction in pain ${ }^{46}$. Neuroimaging studies indicate that upregulation and downregulation of negative emotions are associated with increased activation of prefrontal regions ${ }^{47}$. These observations demonstrate that the PFC plays an important role in pain processing ${ }^{48}$. We found that ONFH patients showed significantly increased ReHo in the dorsolateral prefrontal cortex. Notably, the areas with significantly increased ReHo are in the central part of the PFC. The dorsolateral prefrontal cortex is functionally linked to the descending pain modulation system and has been implicated in top-down pain inhibition, including placebo analgesia ${ }^{49}$. In another orthopedic disease, poor recovery of upper limb pain after surgical interventions for cervical spondylotic myelopathy was found to be associated with the dorsolateral prefrontal cortex ${ }^{50}$. Similarly, during pain onset, a higher BOLD signal response in the dorsolateral prefrontal cortices was observed in fibromyalgia patients than in control subjects ${ }^{51}$. Therefore, brain hyperactivation may be a mechanism underlying the generalized hypervigilance to salient stimuli in pain ${ }^{51}$. 
The sensorimotor cortex is another brain region closely related to the sensory cortex. The sensorimotor cortex includes somatosensory and motor regions and extends to the supplementary motor area (SMA) 52. The FC analysis revealed differences in sensorimotor regions between ONFH patients and controls. These findings are consistent with a previous pain study that showed abnormal functional connectivity of sensorimotor cortex in primary dysmenorrhea patients ${ }^{53}$. Furthermore, some studies have confirmed a relationship between pain persistence and aberrant sensorimotor cortex activity ${ }^{54}$. Interestingly, in the present study, the ONFH patients showed decreased cortical thickness in the supplementary motor cortex. Consistent with this result, a study of diabetic peripheral neuropathy (DPN) with focus on painful DPN revealed impaired motor gray matter in DPN patients ${ }^{55}$. In addition, the duration and frequency of migraine attacks have been found to have strong effects on cortical thickness in the sensorimotor cortex ${ }^{56}$. The increased surface-based ReHo in the premotor cortex observed in the ONFH patients in this study might represent a compensatory increase. In our future work, we aim to explore this topic with a larger sample size. The result that ONFH patients showed significantly increased frontal eye field may indicate the abnormality of visual movement and visual attention network.

\section{Conclusions}

In conclusion, patients with ONFH appear to exhibit cortical and subcortical thinning and abnormal functional activity in specific brain regions associated with sensorimotor and pain processing. This is a pioneer study of the brain mechanisms in ONFH patients, as revealed by comparisons with healthy controls. There are several limitations to note. First, the sample size was small, limiting the statistical power to detect differences. Second, the different stages and types of ONFH were not considered.

\section{List Of Abbreviations}




\begin{tabular}{|ll|}
\hline blood oxygen level dependent & BOLD \\
\hline diabetic peripheral neuropathy & DPN \\
\hline functional connectivity & FC \\
\hline magnetic resonance imaging & MRI \\
\hline osteonecrosis of the femoral head & ONFH \\
\hline prefrontal cortex & PFC \\
\hline regional homogeneity & ReHo \\
\hline regions of interest & ROI \\
\hline resting-state functional magnetic resonance imaging & rs-fMRI \\
\hline supplementary motor area & SMA \\
\hline Total hip arthroplasty & THA \\
\hline Visual Analogue Scale & VAS \\
\hline
\end{tabular}

\section{Declarations}

\section{Ethics approval and consent to participate}

The protocol was approved by the ethics committee of Yueyang Hospital of Integrated Traditional Chinese and Western Medicine. Informed consent was obtained from all study participants.

\section{Consent for publication}

Not applicable.

\section{Availability of data and materials}

The data sets generated and/or analyzed during this study are not publicly available because they contain protected health information. However, deidentified data sets are available from the corresponding authors on reasonable request.

\section{Competing Interests}

The authors declare that they have no competing of interests.

\section{Funding}


This research was funded by the National Key R\&D Program of China (Grant No. 2018YFC2001600); National Natural Science Foundation of China (Grant Nos.: 81802249, 81871836); Shanghai Science and Technology Committee (Grant Nos.: 18511108300, 18441903903900 and 18441903800); Three-Year Action Plan for Traditional Chinese Medicine Development from Shanghai Municipal Health Commission (Grant No.: ZY(2018-2020)-ZWB-1001-CPJS49; ZY(2018-2020)-RCPY-3007); Traditional Chinese Medicine Diagnosis and Treatment Technology Improvement Project from Shanghai Municipal Commission of Health and Family Planning (Grant No.: Zyjx-2017006); and special project of Postgraduate Innovation Training (Grant No.:Y2020021).

\section{Authors' contributions}

J.G. Xu and B. Li: study design, and data acquisition. J. Ma: drafting of manuscript. X.Y. Hua and M.X. Zheng manuscript revision. J.J. Wu and B.B. Huo conducted statistical analyses. X.X. Xing and S.Y. Feng: data collection. All authors read and approved the final manuscript.

\section{Acknowledgments}

We thank all contributors and participants for their contribution to this study.

\section{References}

1. Zalavras CG, Lieberman JR. Osteonecrosis of the femoral head: Evaluation and treatment. J Am Acad Orthop Surg. 2014;22:455-64.

2. Hauzeur JP, Malaise M, de Maertelaer V. A prospective cohort study of the clinical presentation of non-traumatic osteonecrosis of the femoral head: Spine and knee symptoms as clinical presentation of hip osteonecrosis. International orthopaedics. 2016;40:1347-51.

3. Belmar CJ, Steinberg ME, Hartman-Sloan KM. Does pain predict outcome in hips with osteonecrosis? Clinical orthopaedics and related research. 2004:158-162.

4. Zhou W, Qu M, Lv Y, Zhu J. New advances in stem cell therapy for osteonecrosis of the femoral head. Curr Stem Cell Res Therapy. 2019;14:226-9.

5. Lavernia C, D'Apuzzo M, Hernandez VH, Lee DJ. Patient-perceived outcomes in thigh pain after primary arthroplasty of the hip. Clin Orthop Relat Res. 2005;441:268-73.

6. Jo WL, Lee YK, Ha YC, Park MS, Lyu SH, Koo KH. Frequency, developing time, intensity, duration, and functional score of thigh pain after cementless total hip arthroplasty. J Arthroplast. 2016;31:127982.

7. Weiss T, Miltner WH, Liepert J, Meissner W, Taub E. Rapid functional plasticity in the primary somatomotor cortex and perceptual changes after nerve block. Eur J Neurosci. 2004;20:3413-23. 
8. Kamatani D, Hishida R, Kudoh M, Shibuki K. Experience-dependent formation of activity propagation patterns at the somatosensory s1 and s2 boundary in rat cortical slices. Neurolmage. 2007;35:4757.

9. May A. Chronic pain may change the structure of the brain. Pain. 2008;137:7-15.

10. Woo CW, Schmidt L, Krishnan A, Jepma M, Roy M, Lindquist MA, et al. Quantifying cerebral contributions to pain beyond nociception. Nature communications. 2017;8:14211.

11. Morton DL, Sandhu JS, Jones AK. Brain imaging of pain: State of the art. Journal of pain research. 2016;9:613-24.

12. Schmid J, Langhorst J, Gaß F, Theysohn N, Benson S, Engler H, et al. Placebo analgesia in patients with functional and organic abdominal pain: A fmri study in ibs, uc and healthy volunteers. Gut. 2015;64:418-27.

13. Cankaya S, Oktem EO, Saatci O, Velioglu HA, Uygur AB, Ozsimsek A, et al. Paracetamol alters empathy scores in healthy and headache subjects: Functional mri correlates. Journal of clinical neuroscience: official journal of the Neurosurgical Society of Australasia. 2020.

14. Kucyi A, Davis KD. The dynamic pain connectome. Trends in neurosciences. 2015;38:86-95.

15. Farmer MA, Baliki MN, Apkarian AV. A dynamic network perspective of chronic pain. Neurosci Lett. 2012;520:197-203.

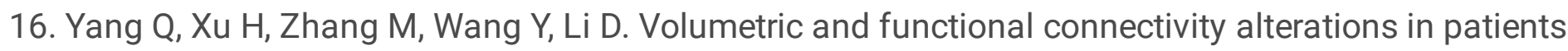
with chronic cervical spondylotic pain. Neuroradiology. 2020.

17. Baliki MN, Mansour AR, Baria AT, Apkarian AV. Functional reorganization of the default mode network across chronic pain conditions. PloS one. 2014;9:e106133.

18. Nigg BM, Liu W. The effect of muscle stiffness and damping on simulated impact force peaks during running. Journal of biomechanics. 1999;32:849-56.

19. Feldman DE, Brecht M. Map plasticity in somatosensory cortex. Science. 2005;310:810-5.

20. De Ridder D, Vanneste S, Van Laere K, Menovsky T. Chasing map plasticity in neuropathic pain. World neurosurgery. 2013;80:901.e901-905.

21. Hellier P, Barillot C, Corouge I, Gibaud B, Le Goualher G, Collins DL, et al. Retrospective evaluation of intersubject brain registration. IEEE Trans Med Imaging. 2003;22:1120-30.

22. Tucholka A, Fritsch V, Poline JB, Thirion B. An empirical comparison of surface-based and volumebased group studies in neuroimaging. Neurolmage. 2012;63:1443-53.

23. Wager TD, Atlas LY, Lindquist MA, Roy M, Woo CW, Kross E. An fmri-based neurologic signature of physical pain. N Engl J Med. 2013;368:1388-97.

24. Zang $Y$, Jiang $T$, Lu Y, He Y, Tian L. Regional homogeneity approach to fmri data analysis. Neurolmage. 2004;22:394-400.

25. Greicius MD, Krasnow B, Reiss AL, Menon V. Functional connectivity in the resting brain: A network analysis of the default mode hypothesis. Proc Natl Acad Sci USA. 2003;100:253-8. 
26. Jiang L, Zuo XN. Regional homogeneity: A multimodal, multiscale neuroimaging marker of the human connectome. The Neuroscientist: a review journal bringing neurobiology neurology psychiatry. 2016;22:486-505.

27. Zuo XN, Xu T, Jiang L, Yang Z, Cao XY, He Y, et al. Toward reliable characterization of functional homogeneity in the human brain: Preprocessing, scan duration, imaging resolution and computational space. Neurolmage. 2013;65:374-86.

28. Dworkin RH, Turk DC, Farrar JT, Haythornthwaite JA, Jensen MP, Katz NP, et al. Core outcome measures for chronic pain clinical trials: Immpact recommendations. Pain. 2005;113:9-19.

29. Yan CG, Wang XD, Zuo XN, Zang YF. Dpabi: Data processing \& analysis for (resting-state) brain imaging. Neuroinformatics. 2016;14:339-51.

30. Li HJ, Cao XH, Zhu XT, Zhang AX, Hou XH, Xu Y, et al. Surface-based regional homogeneity in firstepisode, drug-naïve major depression: A resting-state fmri study. BioMed research international. 2014;2014:374828.

31. Chen X, Hou X, Luo X, Zhou S, Liu X, Liu B, et al. Altered intra- and inter-regional functional connectivity of the anterior cingulate gyrus in patients with tremor-dominant parkinson's disease complicated with sleep disorder. Front Aging Neurosci. 2019;11:319.

32. surgery JO. Chinese guideline for the diagnosis and treatment of osteonecrosis of the femoral head in adults. 2017;9:3-12.

33. neurology BDJBajo. Termination of the central pain pathway in man: The conscious appreciation of pain. 1957;80:606-622.

34. Sandström A, Ellerbrock I, Jensen KB, Martinsen S, Altawil R, Hakeberg P, et al. Altered cerebral pain processing of noxious stimuli from inflamed joints in rheumatoid arthritis: An event-related fmri study. Brain Behav Immun. 2019;81:272-9.

35. Mykland MS, Bjørk MH, Stjern M, Omland PM, Uglem M, Sand T. Fluctuations of sensorimotor processing in migraine: A controlled longitudinal study of beta event related desynchronization. $J$ Headache Pain. 2019;20:77.

36. Kim K, Choi S, Cha M, Lee BH. Effects of mtor inhibitors on neuropathic pain revealed by optical imaging of the insular cortex in rats. Brain research. 2020;1733:146720.

37. Lai KL, Niddam DM, Fuh JL, Chen WT, Wu JC, Wang SJ. Cortical morphological changes in chronic migraine in a taiwanese cohort: Surface- and voxel-based analyses. Cephalalgia: an international journal of headache. 2020;40:575-85.

38. Li J, Yuan B, Li G, Lu X, Guo Y, Yang Y, et al. Convergent syndromic atrophy of pain and emotional systems in patients with irritable bowel syndrome and depressive symptoms. Neurosci Lett. 2020;723:134865.

39. Woodworth DC, Holly LT, Mayer EA, Salamon N, Ellingson BM. Alterations in cortical thickness and subcortical volume are associated with neurological symptoms and neck pain in patients with cervical spondylosis. Neurosurgery. 2019;84:588-98. 
40. Usui C, Kirino E, Tanaka S, Inami R, Nishioka K, Hatta K, et al. Music intervention reduces persistent fibromyalgia pain and alters functional connectivity between the insula and default mode network. Pain medicine (Malden, Mass.). 2020.

41. Ushirozako H, Yoshida G, Togawa D, Omura T, Hasegawa T, Yamato Y, et al. Brain activation in a cynomolgus macaque model of chymopapain-induced discogenic low back pain: A preliminary study. Spine surgery related research. 2019;3:368-76.

42. Makary MM, Polosecki P, Cecchi GA, DeAraujo IE, Barron DS, Constable TR, et al. Loss of nucleus accumbens low-frequency fluctuations is a signature of chronic pain. Proc Natl Acad Sci USA. 2020;117:10015-23.

43. Apkarian AV, Bushnell MC, Treede RD, Zubieta JK. Human brain mechanisms of pain perception and regulation in health and disease. Eur J Pain. 2005;9:463-84.

44. Baliki MN, Petre B, Torbey S, Herrmann KM, Huang L, Schnitzer TJ, et al. Corticostriatal functional connectivity predicts transition to chronic back pain. Nature neuroscience. 2012;15:1117-9.

45. DL M, JS S, research JAJJop. Brain imaging of pain: State of the art. 2016;9:613-24.

46. CA B, Pain JAJ. Meditation experience predicts less negative appraisal of pain: Electrophysiological evidence for the involvement of anticipatory neural responses. 2010;150:428-438.

47. Buhle JT, Silvers JA, Wager TD, Lopez R, Onyemekwu C, Kober H, et al. Cognitive reappraisal of emotion: A meta-analysis of human neuroimaging studies. Cerebral cortex (New York, N. Y.: 1991). 2014;24:2981-2990.

48. AK J, NT H, DM L. CA B, reports WAJCr. Role of functional brain imaging in understanding rheumatic pain. 2012;14:557-567.

49. Hibi D, Takamoto K, Iwama Y, Ebina S, Nishimaru H, Matsumoto J, et al. Impaired hemodynamic activity in the right dorsolateral prefrontal cortex is associated with impairment of placebo analgesia and clinical symptoms in postherpetic neuralgia. IBRO reports. 2020;8:56-64.

50. Sawada M, Nakae T, Munemitsu T, Hojo M. Functional connectivity analysis and prediction of pain relief in association with spinal decompression surgery. World neurosurgery. 2020.

51. Hubbard CS, Lazaridou A, Cahalan CM, Kim J, Edwards RR, Napadow V, et al. Aberrant salience? Brain hyperactivation in response to pain onset and offset in fibromyalgia. Arthritis \& rheumatology (Hoboken, N.J.). 2020.

52. S C, S J, D L, M B, P S, D M, et al. Investigating default mode and sensorimotor network connectivity in amyotrophic lateral sclerosis. 2016;11:e0157443.

53. Han F, Liu H, Wang K, Yang J, Yang L, Liu J, et al. Correlation between thalamus-related functional connectivity and serum bdnf levels during the periovulatory phase of primary dysmenorrhea. Front Hum Neurosci. 2019;13:333.

54. Jenkins L, Chang WJ, Buscemi V, Cunningham C, Cashin A, McAuley JH, et al. Is there a causal relationship between acute stage sensorimotor cortex activity and the development of chronic low back pain? A protocol and statistical analysis plan. BMJ open. 2019;9:e035792. 
55. Zhang Y, Qu M, Yi X, Zhuo P, Tang J, Chen X, et al. Sensorimotor and pain-related alterations of the gray matter and white matter in type 2 diabetic patients with peripheral neuropathy. Hum Brain Mapp. 2020;41:710-25.

56. Magon S, May A, Stankewitz A, Goadsby PJ, Schankin C, Ashina M, et al. Cortical abnormalities in episodic migraine: A multi-center 3t mri study. Cephalalgia: an international journal of headache. 2019;39:665-73.

\section{Figures}
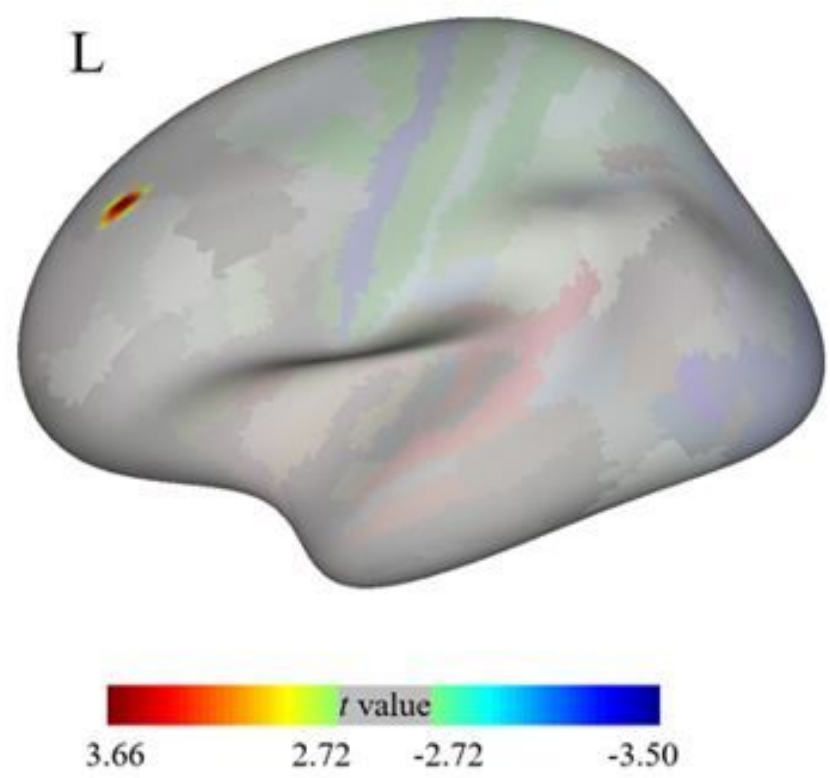
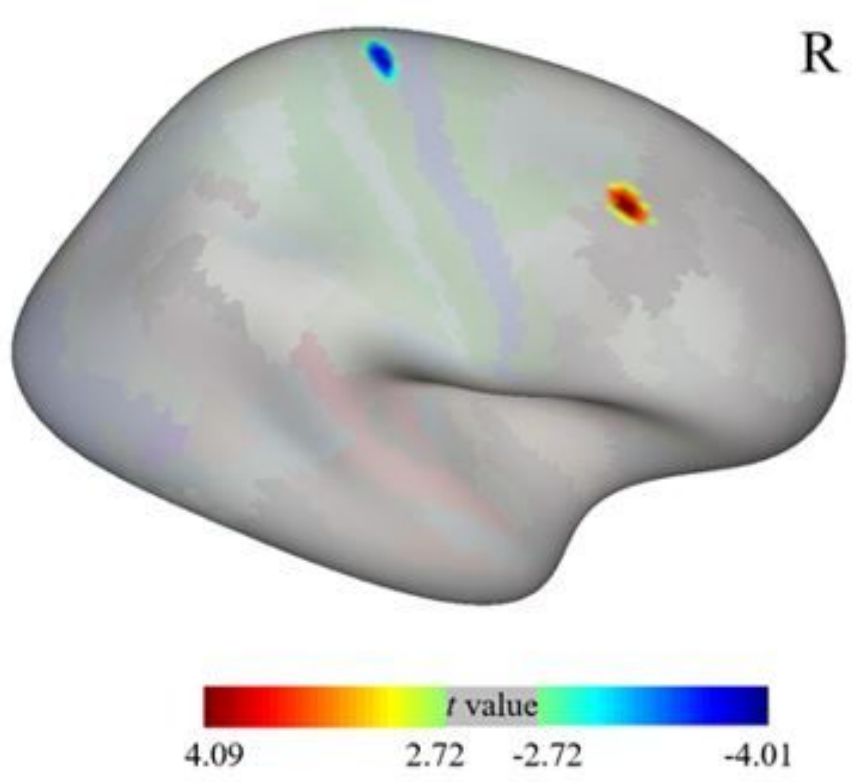

\section{Figure 1}

Comparison of surface-based ReHo values between ONFH patients and healthy controls. Compared to healthy controls, ONFH patients showed significantly increased surface-based ReHo in areas distributed mainly in the left dorsolateral prefrontal cortex and frontal eye field, the right frontal eye field and premotor cortex and decreased surface-based ReHo in the right primary motor cortex and primary sensory cortex. 

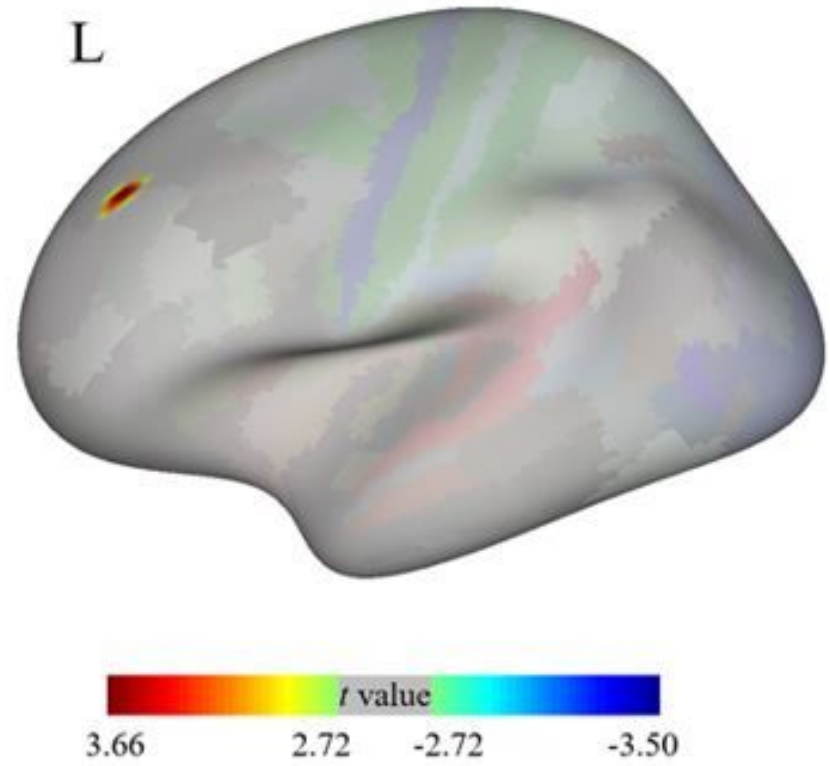

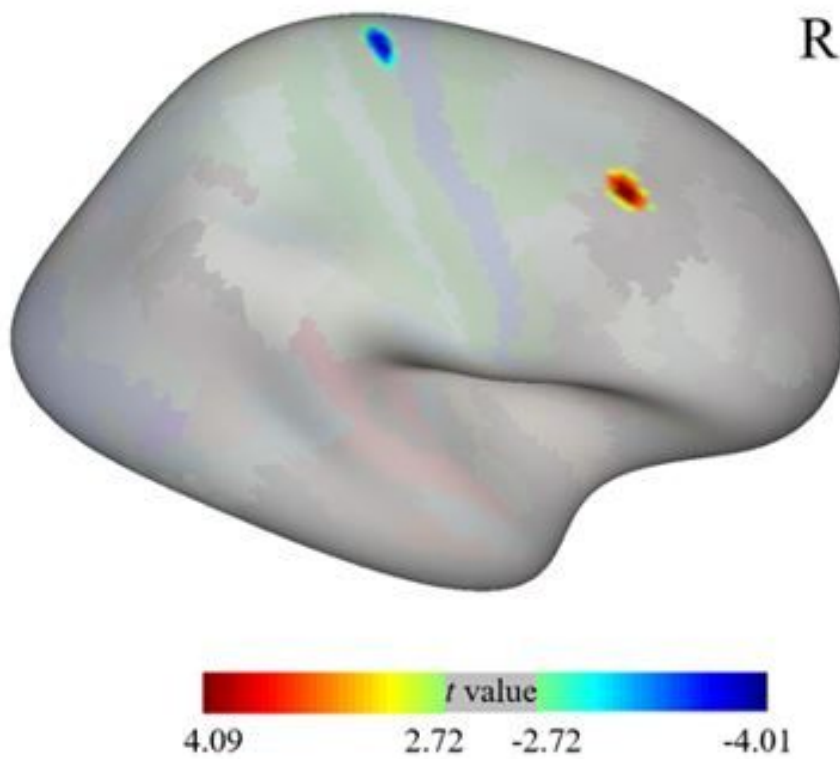

\section{Figure 1}

Comparison of surface-based ReHo values between ONFH patients and healthy controls. Compared to healthy controls, ONFH patients showed significantly increased surface-based ReHo in areas distributed mainly in the left dorsolateral prefrontal cortex and frontal eye field, the right frontal eye field and premotor cortex and decreased surface-based ReHo in the right primary motor cortex and primary sensory cortex.

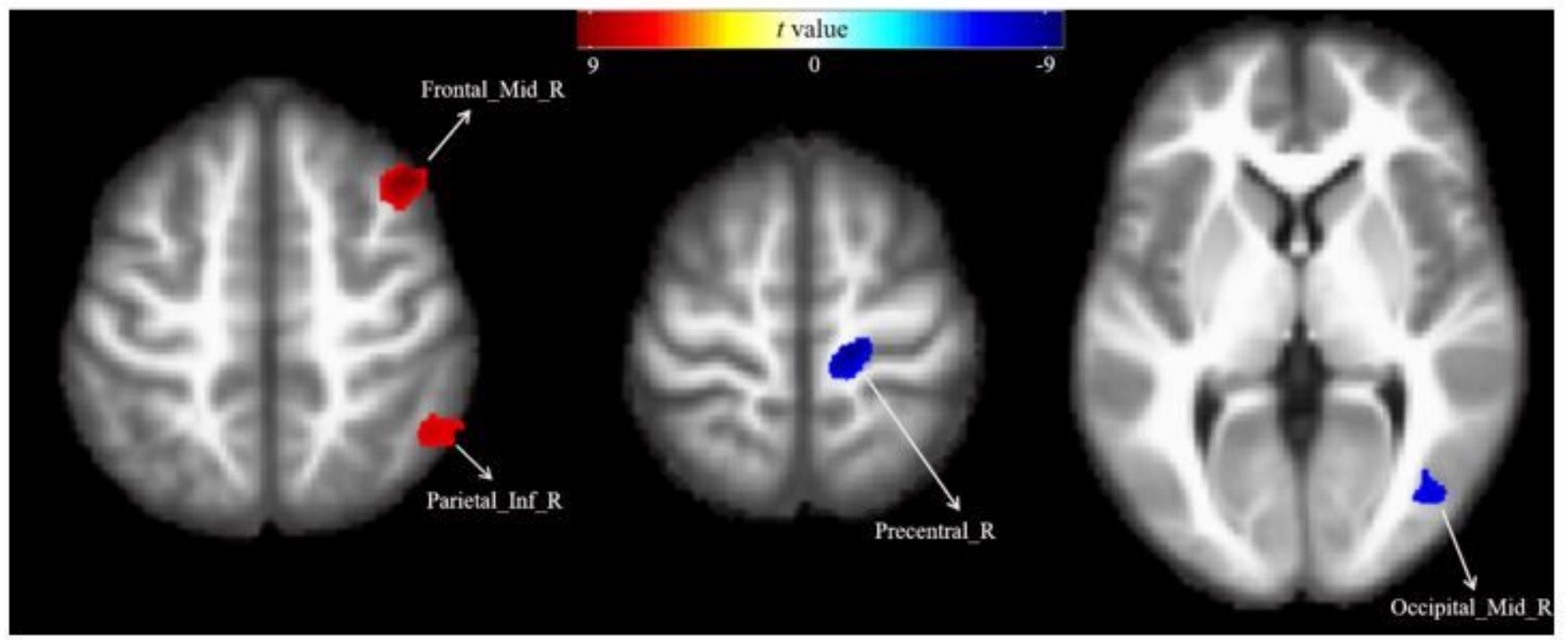

\section{Figure 2}

Comparison of FC values between ONFH patients and healthy controls When the area with decreased surface-based ReHo in the frontal eye field and right premotor cortex was used as ROI, the ONFH patients displayed increased FC in the right middle frontal cortex and right inferior parietal cortex and 
decreased FC in the right precentral cortex and right middle occipital cortex. Frontal_Mid_R: right middle frontal gyrus; Parietal_Inf_R: right inferior parietal gyrus; Precentral_R: right precentral gyrus;

Occipital_Mid_R: right middle occipital gyrus

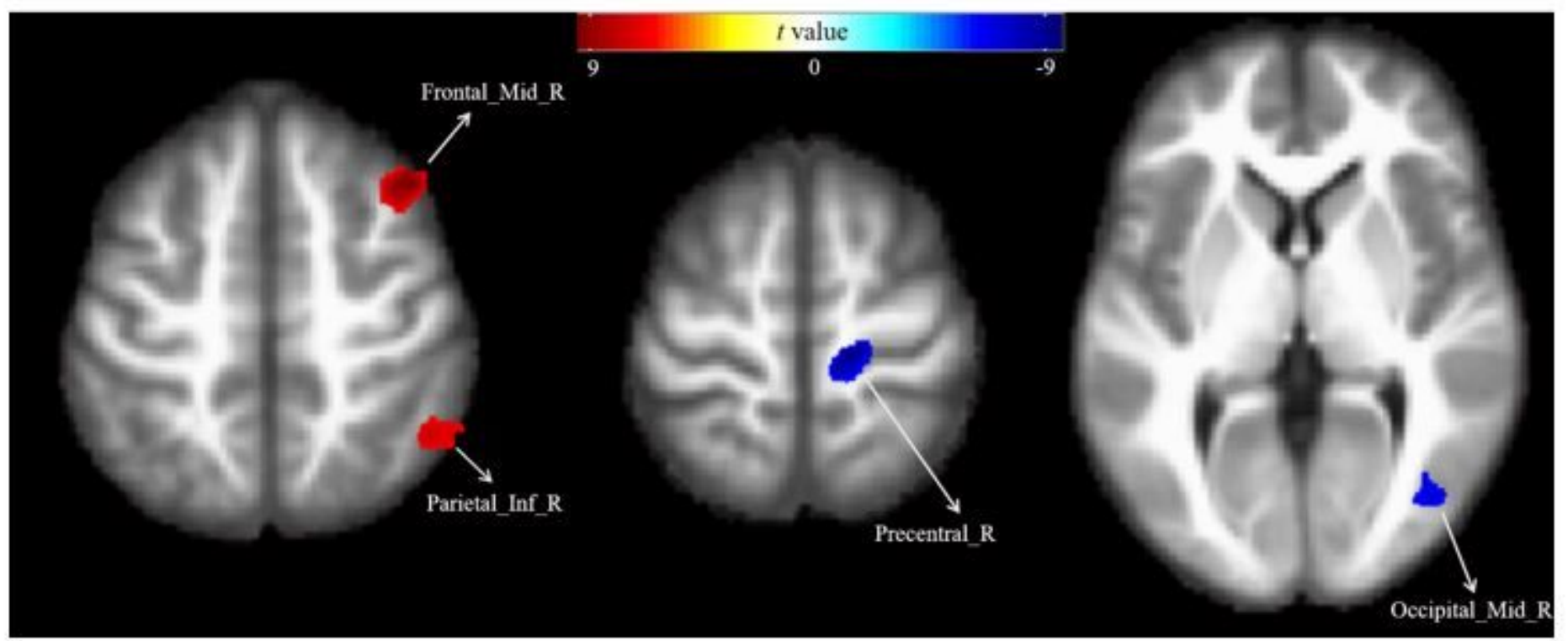

Figure 2

Comparison of FC values between ONFH patients and healthy controls When the area with decreased surface-based ReHo in the frontal eye field and right premotor cortex was used as ROI, the ONFH patients displayed increased FC in the right middle frontal cortex and right inferior parietal cortex and decreased FC in the right precentral cortex and right middle occipital cortex. Frontal_Mid_R: right middle frontal gyrus; Parietal_Inf_R: right inferior parietal gyrus; Precentral_R: right precentral gyrus;

Occipital_Mid_R: right middle occipital gyrus

a

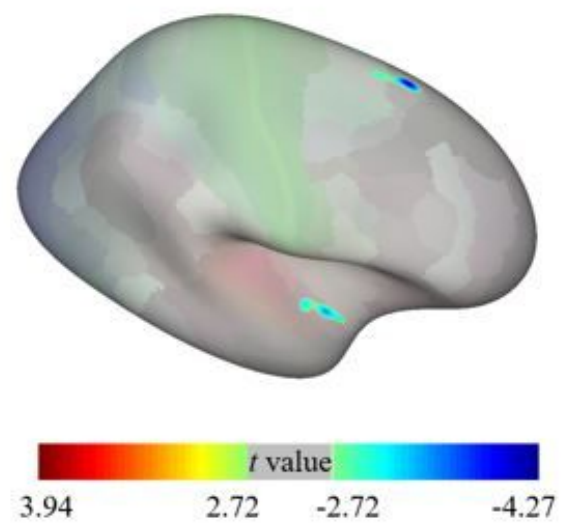

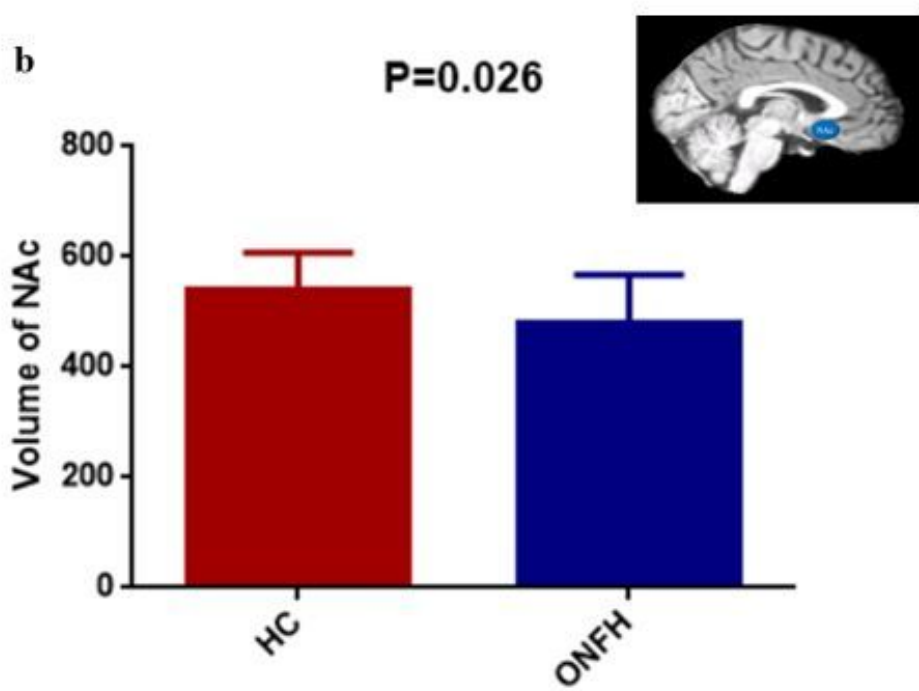

Figure 3 
Comparison of structural images between ONFH patients and healthy controls. a: Comparison of cortical thickness; b: Comparison of volume of subcortical gray matter nuclei. ONFH patients showed significantly decreased cortical thickness in areas mainly distributed in the para-insular area, posterior insular area, anterior superior temporal area, frontal eye field and supplementary motor cortex. In addition, comparison of the volume of subcortical gray matter nuclei between the two groups revealed significantly decreased values in the right nucleus accumbens in ONFH patients compared with healthy controls. NAc: nucleus accumbens

a

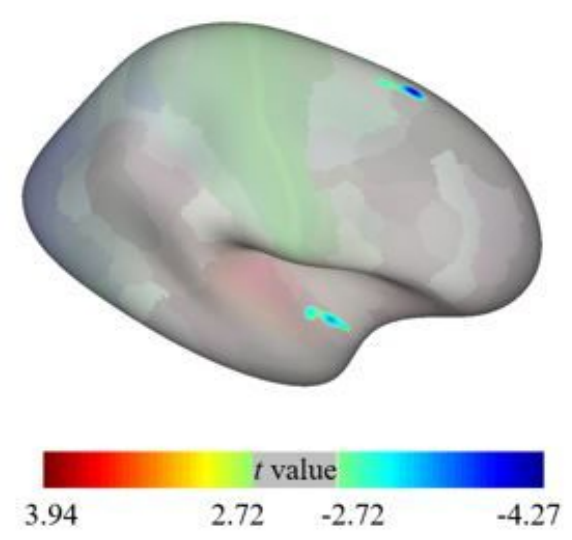

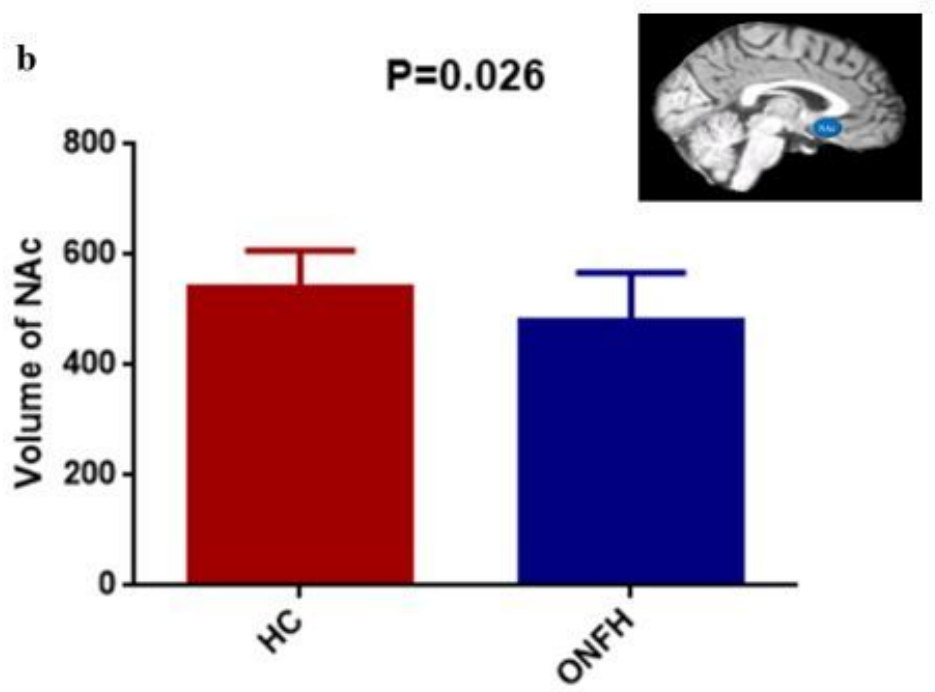

Figure 3

Comparison of structural images between ONFH patients and healthy controls. a: Comparison of cortical thickness; b: Comparison of volume of subcortical gray matter nuclei. ONFH patients showed significantly decreased cortical thickness in areas mainly distributed in the para-insular area, posterior insular area, anterior superior temporal area, frontal eye field and supplementary motor cortex. In addition, comparison of the volume of subcortical gray matter nuclei between the two groups revealed significantly decreased values in the right nucleus accumbens in ONFH patients compared with healthy controls. NAc: nucleus accumbens 